\title{
TUMOR DO GLOMO JUGULAR
}

\author{
antonio Cesar G. Borges * \\ Mario Ferreira Coutinho** \\ ADÃO ANICET *** \\ Décio LisBoa CASTRO *****
}

Os tumores do glomo jugular são pouco comuns e geralmente se iniciam no ouvido médio, dando sintomas otológicos ${ }^{18}$. O neurologista toma contato com esta patologia quando, em estágios mais avançađos, a neoplasia penetrou no interior da cavidade craniana, dando sintomas de comprometimento do sistema nervoso central ou de nervos cranianos. A raridade desta entidade nosológica e a fossivel semelhança com o neuroma do acústico, levou-nos a relatar este caso de tumor de glomo jugular.

As formações glômicas foram inicialmente descritas por Guild ${ }^{12}$ que as localizou junto dos nervos de Arnold (ramo auricular do vago) e de Jacobson (ramo timpânico do glossofaríngeo); mais da metade destes grupamentos celulares estavam na adventicia do golfo da jugular. A inervação dessas estruturas se faz pelos nervos adjacentes e, a vascularização, pela artéria faringea ascendente.

Alexander e col. ${ }^{1}$ referem que, além destas situações, grupos celulares semelhantes são encontrados no gânglio nodoso do vago, adventícia da aorta asçndente, tronco arterial inominado, provavelmente na artéria pulmonar, assim como na órbita, junto do gânglio ciliar. Outras regiōes fora do crânio podem ser sede de estruturas glômicas como, por exemplo, a laringe e o estômago ${ }^{14}$.

Do ponto de vista histológico, Muler e col. ${ }^{20}$ descrevem o glomo jugular como constituído de duas porções embriologicamente distintas: uma de origem mesodérmica, muito vascularizada, que lhe confere a denominação de glomo; a outra, ectodérmica, nervosa, não cromafínica, possivelmente de função endócrina. A primeira é pressotermorreguladora e a segunda asseguraria ao feto, por via hormonal, as funções que, na vida extrauterina, são garantidas pelos sistemas simpático e parassimpático. Admite-se que é sensivel às modificações de $\mathrm{pH}$ e $\mathrm{pCO}_{2}$ da circulação cerebral.

Rosenwasser (citado por Siekert ${ }^{24}$ ) foi quem primeiro relatou um caso de tumor de glomo jugular. A partir dai, maior número de casos passou a ser descrito. Dockerty e col. ${ }^{11}$ atribuem a raridade da neoplasia deste tipo

Trabalho realizado no Serviço de Neurologia e Neurocirurgia do Hospital da Sociedade Portuguesa de Beneficência (Porto Alegre), apresentado no IX Congresso Brasileiro de Neurocirurgia (julho de 1972, Rio de Janeiro): * Médico residente; ** Neurocirurgião-chefe; *** Neuro-radiologista; **** Otologista. 
à confusão diagnóstica com hemangioendoteliomas, endoteliomas e polipos angiomatosos do ouvido médio.

Alford e Guilford ${ }^{2}$ referiram serem estes tumores de evolução lenta, usualmente benignos, e predominantes em mulheres de meia idade. Geralmente não são funcionantes. Porém, recentemente foi verificada a presença de norepinefrina em paraganglioma não cromafinico de glomo jugular em paciente negra com hipertensão arterial grave ${ }^{8}$. Existem associações de quemodectomas jugular e carotídeo, jugular e retroperitoneal, assim como uma tendência hereditária-familiar ${ }^{22}$.

As manifestações clínicas do tumor de glomo jugular variam na dependência do ponto de origem da neoplasia e da direção que a mesma assume. Os quemodectomas que se iniciam no ouvido médio podem se dirigir para baixo, fazendo saliência na face lateral do pescoço ou na faringe ou, ainda, penetrar na cavidade craniana, alargando o forame rasgado posterior, comprometendo os nervos cranianos ou determinando sintomas de hipertensão intracraniana. Ambas as possibilidades são raras. O tumor ainda pode dirigir-se para o tímpano, exteriorizando-se no canal auditivo externo ou para a mastóide, erosando-a e simulando aneurisma cirsóide da pele retroauricular.

$\mathrm{Na}$ série de Alford e Guilford ${ }^{2}$ os sinais e sintomas mais freqüentes, em ordem decrescente de incidência, foram surdez de percepção, zumbidos, paralisia facial periférica e otorréia. A presença de massa polipóide no interior do conduto auditivo externo ocorreu em $56 \%$ dos casos. O comprometimento dos nervos cranianos, assim como sinais de lesão cerebelar ou bulbopontina são mais raros (em torno de $20 \%$ dos 316 casos da série de Alford e Guilford). Embora as manifestações neurológicas sejam tardias, Siekert ${ }^{24}$ afirma que, ocasionalmente, podem ser precoces (lesão inicial do nervo facial ou de um dos nervos do forame jugular, especialmente o vago). Poppen (citado por Henson e col. ${ }^{13}$ ) relatou um caso de tumor de glomo jugular dando sinais de compressão bulbar.

OB SER VAÇ A

A.J.R., sexo masculino, 57 anos de idade, admitido em 20 de setembro de 1967 (Reg. n. ${ }^{\circ}$ 1658) relatando que há 4 anos notou zumbidos à direita que progressivamente se tornaram contínuos. Há 2 anos não ouve pelo ouvido direito; paralisia facial periférica direita há 20 dias. Exame neurológico — Dismetria à direita pela prova index-nariz; nistagmo horizontal com componente rápido para a direita; paralisia facial periférica à direita; desvio do véu do paladar para a esquerda; refluxo de líquidos pelo nariz. Exame audiológico - Anacusia à direita e presença de massa polipóide no interior do conduto auditivo externo. Craniogramas (incidências anteroposterior, perfil, Towne, Stenvers e Hirtz) - Extensa osteólíse na região da pirâmide petrosa à direita, com rotura da cortical superior, estendendo-se ao ouvido interno e destruindo totalmente a cóclea e parcialmente a mastóide e a janela oval. A caixa timpânica também foi atingida e havia esclerose da mastóide direita (fig. 1, A). Pneumencefalografia fracionada - Quarto ventriculo, aqueduto de Sylvius e porção posterior do terceiro ventriculo sem desvios ou deformações; a cisterna pontocerebelosa direita estava afastada $10 \mathrm{~mm}$ do plano ósseo e deformada em arco de contôrno regular. Havia também dilatação da cisterna ambiens do mesmo lado e a presença de cavo comunicante do septo pelúcido (Fig. $1, B$ e C). Angiografias carotidea e vertebral direitas não mostraram anormalidades. Biópsia através do conduto auditivo externo: existência de células epitelióldes com núcleos 
uniformes e citoplasma eosinofílico, dispostas em alvéolos, em meio a estroma marcadamente rico em capilares. O diagnóstico histopatológico foi de tumor de glomo jugular (paraganglioma não cromafínico).

Intervenção cirúrgica - Por via translabirintica foi extirpado parcialmente o tumor que ocupava toda a caixa timpânica e a parte petrosa do temporal, atingindo a sua porção póstero-superior. Foi feita a descompressão do nervo facial que estava conservado. Após a intervenção o paciente foi submetido a radioterapia. Seis meses após mostrava paralisia facial em regressão e conseguia fechar a pálpebra direita.
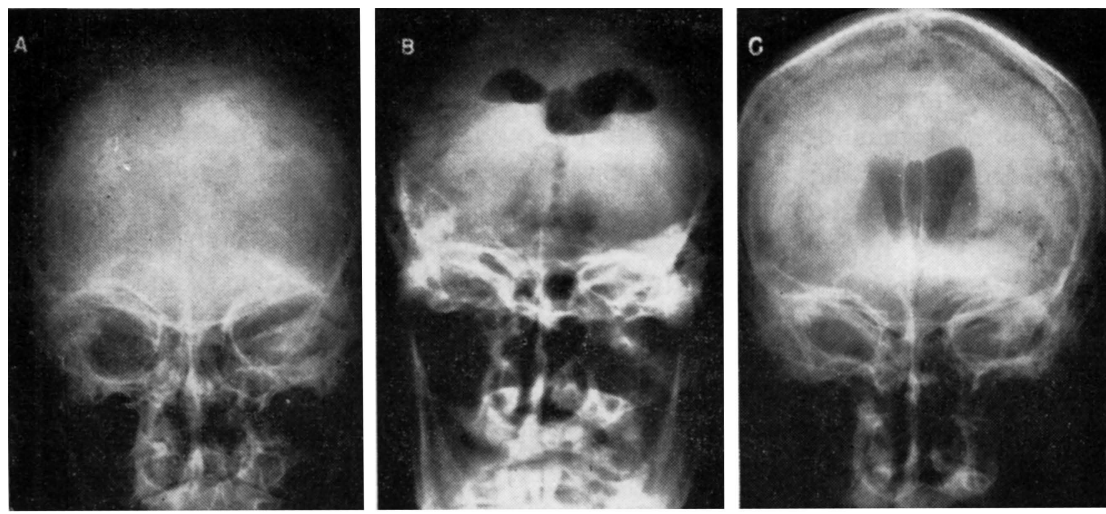

Fig. 1 - Caso A.J.R. Em A, osteólise da porção petrosa do osso temporal direito, rotura da cortical superior; em $B$, cisterna pontocerebelosa direita afastada do plano ósseo e deformada em arco de contorno regular, além de dilatação da cisterna ambiens do mesmo lado; em $C$, cavo comunicante do septo pelúcido.

\section{COM E N T Ã R I S}

A patologia mais freqüente que acomete o osso temporal é o neuroma do acústico que, embora alargue o conduto auditivo interno, não costuma destruir a porção petrosa do osso temporal. Os exames radiológicos são de capital importância na investigação de tais lesões ${ }^{5}, 16,23$, pois as alterações predominantes na presença do tumor glômico são a erosão da porção petrosa do temporal, apagamento ou erosão da mastóide e, em menor número de casos, o alargamento do forame jugular. Quando ex:ste apenas esclerose da mastóide é difícil separar tumor de mastoidite ${ }^{24}$. As modificações ósseas podem ser detectadas nas incidências radiológicas para o estudo da base do crânio e osso temporal. A tomografia auxilia a diferenciar tumor timpânico, do jugular ou misto.

A pneumencefalografia fracionada permite o estudo das cisternas da fossa posterior, deformadas pelo processo expansivo. A tomografia associada a este método mostra mais detalhes das estruturas desta região ${ }^{3}$. Nos volumosos tumores do nervo acústico, a cisterna ambiens e a cisterna bulbar do mesmo lado do tumor tornam-se dilatadas e podem não ser observadas no lado são. O quarto ventrículo pode estar deslocado para trás e para o lado oposto à neoplasia. 
O tumor de glomo, quando se inicia na adventícia da jugular, comprime as estruturas que cursam o forame rasgado posterior, modificando a forma de abertura deste canal no endocrânio. Se os sinais forem precoces, é possível o diagnóstico da neoplasia da parte nervosa ou da parte vascular do canal, tais como neuromas do IX, X e XI nervos cranianos ou malformações vasculares. Com a progressão da doença ao exocrânio, faz-se necessário afastar o carcinoma do faringe ou da cavidade timpânica, meningeomas, condromas e, até mesmo, aneurismas da carótida (Guirguis, citado por Di Chiro e col. ${ }^{9}$ ). Estão descritos na literatura casos de tumor de glomo jugular simulando meningeomas da fossa média ${ }^{17}$ ou da fossa posterior ${ }^{6,11}$. E preciso ressaltar que as metástases podem dar quadro clínico e radiológico de tumor de glomo jugular; daí a importância da biópsia antes da indicação da radioterapia ${ }^{21}$. Angiografias por via carotídea e vertebral, além de delinear o tumor, são úteis para afastar as fístulas júgulo-carotídeas em associação com o tumor ou, ainda, as malformações vasculares do forame jugular ${ }^{4}, 10$. Henson e col. ${ }^{13}$ atribuem a freqüente falha da angiografia para detectar esses tumores ao suprimento sangüíneo pela carótida externa.

Alford e Guilford ${ }^{2}$, reunindo dados clínicos e radiológicos, classificaram os tumores glômicos em estágios de 0 a 4 em graus crescentes de gravidade. Até o estágio 2 (surdez, polipo no conduto auditivo externo, paralisia facial e alargamento ou não do forame jugular) deve ser praticada a timpanotomia ou a mastoidectomia, respectivamente para tumores pequenos e grandes. $\mathrm{Em}$ fases mais avançadas, a cirurgia tem fornecido resultados insatisfatórios $6,11,13$, embora tenham sido relatados casos em que a cirurgia permitiu remoção de toda a neoplasia ${ }^{19}, 25$. Para o estágio 3 em que há erosão da parte petrosa e comprometimento de nervos cranianos além do VII e VIII, Alford e Guilford ${ }^{2}$ aconselham a radioterapia inicial, que pode ser de grande valor, quer isoladamente ou associada à cirurgia ${ }^{10}$. Como a radioterapia não é livre de riscos, Kempe e col. ${ }^{15}$ publicaram recentemente trabalho sobre a técnica de ablação de tais tumores. Quando há hipertensão intracraniana são sugeridas, por muitos autores, intervenções descompressivas da fossa posterior ou o emprego de válvulas para correção de hidrocéfalo, quando este existir.

\section{R E S U M O}

É relatado um caso de tumor de glomo jugular com 4 anos de evolução. Os exames neuro-radiológicos mostraram destruição da pirâmide petrosa direita, deslocamento da cisterna pontocerebelosa direita e dilatação da cisterna ambiens do mesmo lado. $O$ paciente foi submetido à cirurgia otológica, sendo o tumor removido parcialmente. Após radioterapia houve remissão parcial dos sintomas.

S U M M A R Y

Tumor of the glomus jugulare. A case report.

A case of tumor of the glomus jugulare in a 57 years old patient in which the symptoms lasted four years is reported. The neuroradiological 
examination showed erosion of the petrous part of the right temporal bone, displacement of the right pontocerebellar cistern and dilatation of the ambiens cistern in the same side. The patient was submitted to otological surgery the tumor being partially removed. After the irradiation therapy some symptoms disappeared.

\section{R E F E R E N I A S}

1. ALEXANDER, E. Jr.; BEAMER, P. R. \& WILLIAMS, J. O. - Tumor of the glomus jugulare with extension into the middle ear (nonchromaffin paraganglioma or carotid-body-type tumor). J. Neurosurg. 8:515, 1951.

2. ALFORD, B. R. \& GUILFORD, F, R. - A comprehensive study of tumors of the glomus jugulare. Laryngoscope 72:765, 1962.

3. ANICET, A. \& WANDERLEY, C. - Diagnóstico radiológico do neuroma do acústico. Anais do XVIII Congresso Brasileiro de Otorrinolaringologia (Porto Alegre, 24 a 29 de outubro de 1969).

4. BATSAKIS, J. G. \& FARBER, E. R. - Chemodectomas of the head and neck. Michigan Med. Center J. 33:124, 1967.

5. CERVINO, H. O.; CHOUZA, C.; SAZBON, L. \& AGUERRE, E. - Chemodectomas de los glomus yugular y carotideo. Acta Neurol. Latinoamer. 15:33, 1969.

6. CHAMBERS, W. R. - Tumor of the glomus jugulare resembling brain tumor. Jour. Internat. Coll. Surg. 22:691, 1954.

7. Correlation conferences in radiology and pathology. Glomus jugulare tumor. New York State. J. Med. 1:1661, 1970.

8. DE LELLIS, R. A. \& ROTH, J. A. - Norepinephrine in a glomus jugulare tumor. Histochemical demonstration. Arch. Pathol. 92:73, 1971.

9. DI CHIRO, G.; FISHER, R. L. \& NELSON, K. B. - The jugular foramen. J. Neurosurg. 21:447, 1964.

10. DIEPEVEEN, J.; HENTZER, E. \& ROVSING, H. - Non-chromaffin paragangliomas. Brief review and clinical radiological follow-up on nine cases. Acta Otolaryng. (Stockholm) 68:142, 1969.

11. DOCKERTY, M. B.; LOVE, J. G. \& PATTON, N. M. - Nonchromaffin paraganglioma of the middle ear: report of a case in which the clinical aspects were those of a brain tumor. Proc. Mayo Clin. 26:25, 1951.

12. GUILD, S. R. - The glomus jugulare: a nonchromaffin paraganglion in man. Ann. Otol. Rhin. Laryng. 62:1045, 1953.

13. HENSON, R. A.; CRAWFORD, J. V. \& CAVANAGH, J. B. - Tumors of the glomus jugulare. J. Neurol. Neurosurg. Psychiat. 16:127, 1953.

14. ISHIDA, M.; HASEGAWA, S.; SATO, T. \& TATEISHI, R. - Glomus tumor (nonchromaffin paraganglioma) of the larynx. Laryngoscope 81:957, 1971.

15. KEMPE, L. G.; VANDERARK, G. D. \& SMITH, D. R. - The neurosurgical treatment of the glomus jugulare tumors. J. Neurosurg. 35:59, 1971.

16. KIM, S. K. \& CAPP, M. P. - Jugular foramen and early roentgen diagnosis of glomus jugulare tumor. Amer. J. Roentg. 97:597, 1966.

17. KRUSE Jr., F. - Petrous ridge chemodectoma (nonchromaffin paraganglioma) simulating meningioma. J. Neurosurg. 17:1088, 1960.

18. LE COMPTE, P. M. - Tumors of the carotid body and related structures (chemoreceptor system). In Atlas of Tumor Pathology. National Research Council, Washington D.C., 1951.

19. MEACHAM, W. F. \& CAPPS, J. M. - Intracranial glomus jugulare tumor with successful surgical removal. J. Neurosurg. 17:157, 1960.

20. MULER, H.; COTIN, G. \& PRACHE, H. - Le glomus jugulaire et ses tumeurs. Annales d'Oto-laryngol. et Chirur. cervicofaciale $87: 225,1970$.

21. MULER, H.; COTIN, G.; PRACHE, H. \& DESBLED, M. - Glomus et faux glomus. Annales d'Oto-laryngol. et Chirur. cervicofaciale 87:295, 1970.

22. RESLER, D. R.; SNOW, J. B. Jr. \& WILLIAMS, G. R. - Multiplicity and familial incidence of the carotid body and glomus jugulare tumors. Ann. Otol. Rhinol. Lar. 75:114, 1966 
23. RIEMENSCHNEIDER, P. A.; HOOPLE, G. D.; BREWER, D.; JONES, D. \& DECKER, A. - Roentgenographic diagnosis of tumors of the glomus jugularis. Amer. J. Roentg. 69:59, 1963.

24. SIEKERT, R. G. - Neurological manifestations of tumors of the glomus jugulare. Arch. Neurol. Psychiat. (Chicago) 76:1, 1956.

25. THOMS, O. J.; SCHAW, D. T. \& TROWBRIDGE, W. V. - Glomus jugulare tumor. Report of a case with surgical removal. J. Neurosurg. 17:500, 1960.

Serviço de Neurologia e Neurocirurgia - Hospital da Sociedade Portuguesa de Beneficência — Av. Independência 270 - 90000 Porto Alegre, RS - Brasil. 